\title{
NOVEMBRE 1971
}

53-ANNEE-N・ 635

\section{revue internationale de la croix-rouge}

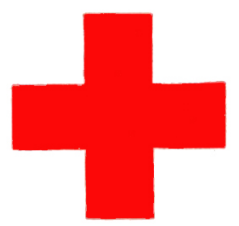

INTER ARMA CARITAS

GENËVE

COMITÉ INTERNATIONAL DE LA CROIX-ROUGE FONDÉ EN 1863 


\section{COMITÉ INTERNATIONAL DE LA CROIX-ROUGE}

MM. MARCEL A. NAVILLE, président (membre depuis 1967)

JEAN PICTET, docteur en droit, président de ia Commission juridique, vice-président (1967)

HARALD HƯBER, docteur en droit, juge fédéral, vice-president (1969)

PAUL RUEGGER, ambassadeur, président du CICR de 1948 a 1955 (1948)

GUILLAUME BORDIER, ingénieur dipl. E.P.F., M.B.A. Harvard, banquier (1955)

HANS BACHMANN, docteur en droit, Stadtrat de Winterthour (1958)

JACQUES FREYMOND, docteur ès lettres, directeur de l'Institut universitaire de hautes études internationales, professeur à l'Université de Genève (1959)

DIETRICH SCHINDLER, docteur en droit, professeur à l'Université de Zurich (1961)

M"He MARJORIE DUVILLARD, infirmière (1961)

MM. MAX PETITPIERRE, docteur en droit, ancien président de la Confédération suisse (1961)

ADOLPHE GRAEDEL, député au Conseil national suisse de 1951 à 1963, ancien socrétaire général de la Fédération internationale des ouvriers sur métaux (1965)

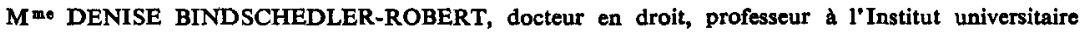
de hautes études internationales (1967)

MM. JACQUES F. DE ROUGEMONT, docteur en médecine (1967)

ROGER GALLOPIN, docteur en droit, ancien directeur général (1967)

WALDEMAR JUCKER, docteur en droit, secrétaire de l'Union syndicale suisse (1967)

VICTOR H. UMBRICHT, docteur en droit, administrateur (1970)

PIERRE MICHELI (1971)

Membres honoraires: M. JACQUES CHENEVIËRE, vice-president d'honneur; Mil LUCIE ODIER, vice-présidente d'honneur; MM. CARL J. BURCKHARDT, PAUL CARRY, $M^{\text {me }}$ MARGUERITE GAUTIER-VAN BERCHEM, MM. SAMUEL A. GONARD, EDOUARD DE HALler, PAUl LOGOZ, RODOLFO OLGIATI, FRÉdériC SIORDET, ALFREDO VANNOTTI, ADOLF VISCHER.

Direction: MM. JEAN-LOUIS LE FORT, secrétaire général;

RAYMOND COURVOISIER, assistant spécial du président et directeur du département des opérations;

CLAƯDE PILLOƯD, directeur du département des principes et du droit. 


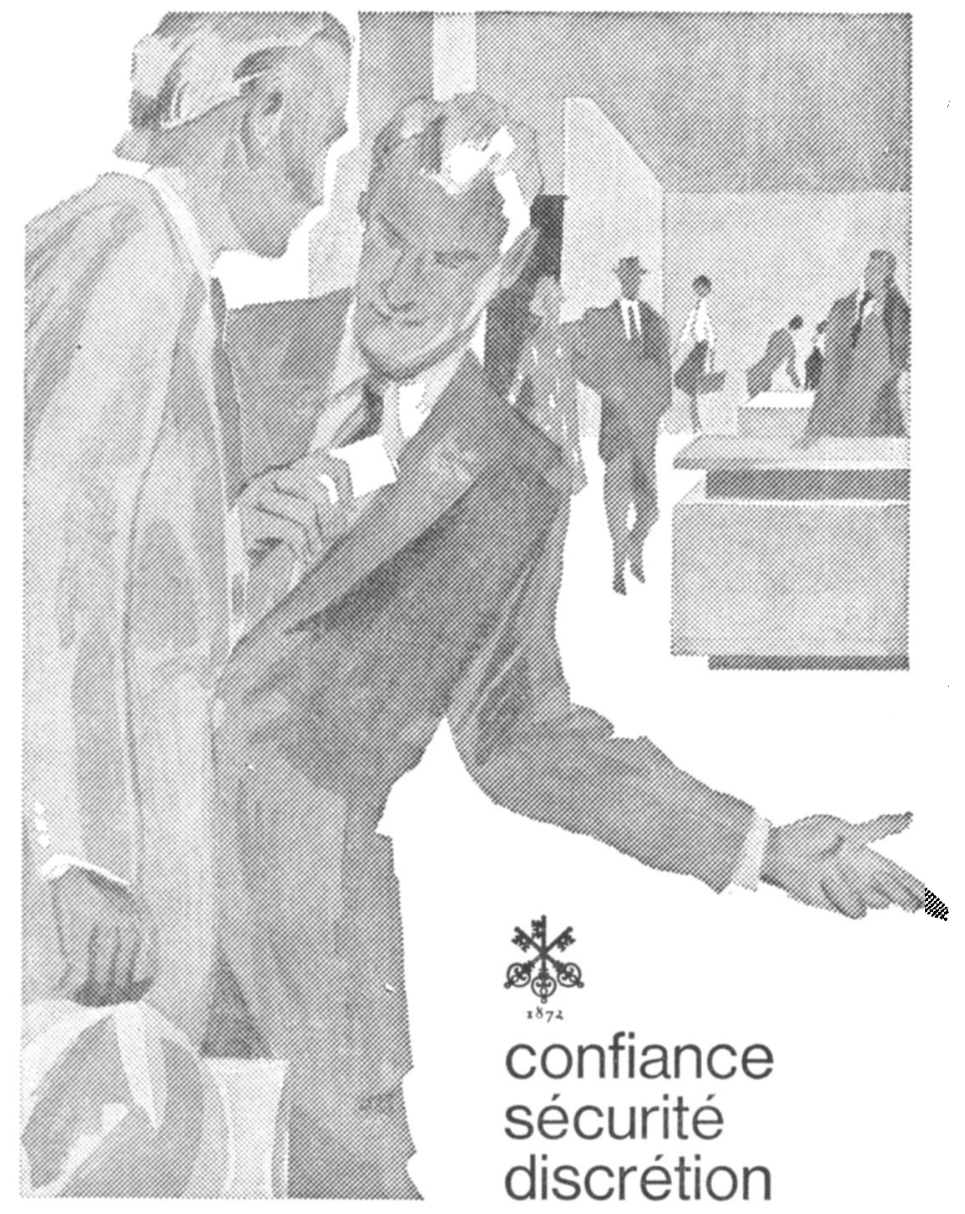

\section{SOCIÉTÉ DE BANQUE SUISSE SWISS BANK CORPORATION}

Siège de Genève: 2, rue de la Confédération Tél. 266211 


\section{SOCIETE FIDUCIAIRE ROMANDE OFOR S.A.}

Place Saint-Gervais 1, Genève - Tél. 317050

४ Expertises, revisions et organisations comptables

\ Domiciliation et administration de sociétés

$\downarrow$ Assainissements et liquidations

— Interventions et conseils en matière fiscale

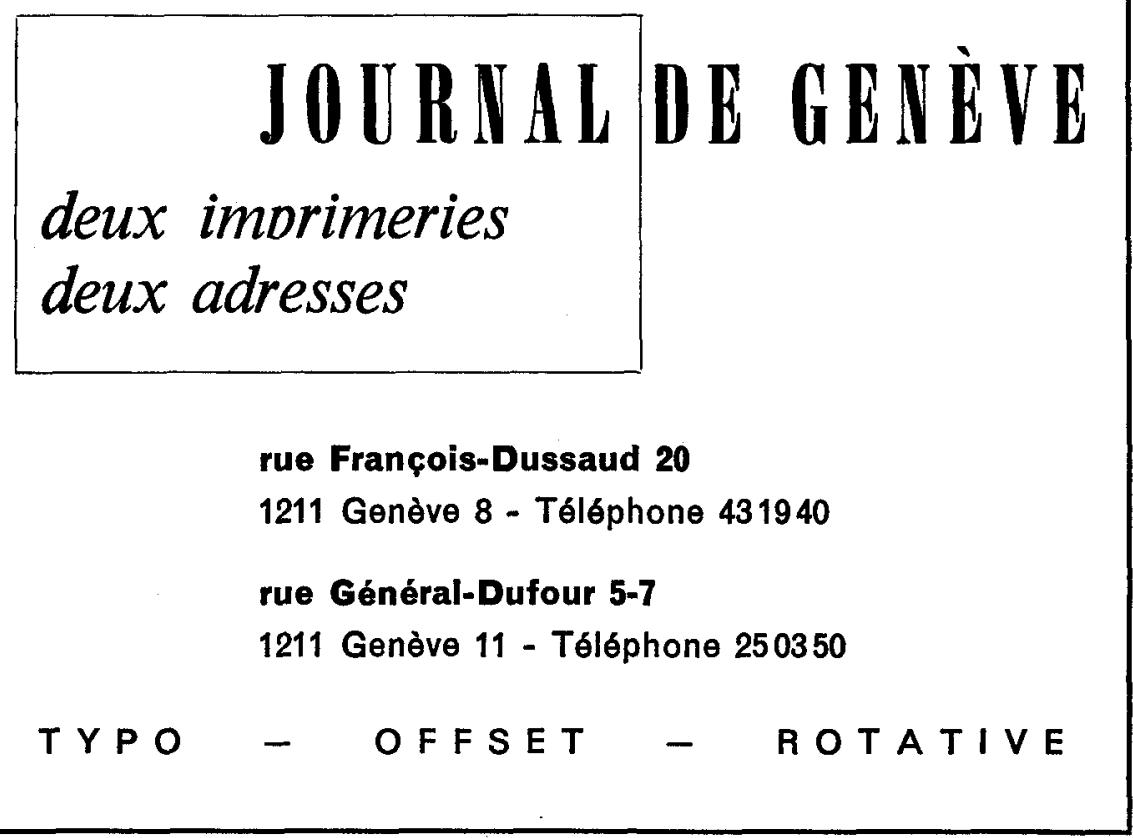




\section{SOMMAIRE}

COMITE INTERNATIONAL DE LA CROIX-ROUGE
DANS LE MONDE dE LA CROIX-ROUGE

FAITS ET DOCUMENTS

\section{REVUE INTERNATIONALE \\ DE LA CROIX-ROUGE}

NOVEMBRE 1971 - N 635

Conférence d'experts gouvernementaux -24 mai12 juin 1971 (II) . . . . . . . . . . . . . 661

Activités extérieures:

Afrique - République du Vietnam - République khmère - Ceylan - Japon - Bolivie - Irlande du Nord - Moyen-Orient - République arabe du Yémen - République démocratique populaire du Yémen

A Genève:

L'empereur et l'impératrice du Japon au CICR . 684 Expériences pseudo-médicales . . . . . . . . . 684

Vers la deuxième session de la Conférence d'experts gouvernementaux . . . . . . . . . 686

Une Agence de recherches à Dacca . . . . . . 687

Le service de radiodiffusion de la Croix-Rouge 689

Secours distribués ou transmis par le CICR durant l'année 1970 . . . . . . . . . . . . . 691

Institut régional de formation pour l'Afrique occidentale . . . . . . . . .

Institut latino-américain de formation de la CroixRouge . . . . . . . . . . 695

Le Fonds de l'Impératrice Shôken . . . . . . . 700

Protection des journalistes en mission périlleuse dans les zones de conflit armé

Conférence sur la mise hors-la-loi des armes biologiques, chimiques et nucléaires . . . . .

Colloque international sur les règles humanitaires et instructions militaires . . . . . . . . .

$\mathrm{X}^{\mathrm{e}}$ Assemblée internationale d'« Amnesty International " . . . . . . . . . . . . . . . . . . . 708

Conférence interparlementaire. . . . . . . . . . 709

Secours à l'enfance . . . . . . . . . . . 710

Les organisations bénévoles et le développement 712 


\section{INTERNATIONAL \\ REVIEW \\ OF THE RED CROSS}

\section{SUPPLEMENTS \\ DE LA REVUE}

EN LANGUE ESPAGNOLE

EN LANGUE ALLEMANDE

\section{REVUE INTERNATIONALE DE LA CROIX-ROUGE}

Une édition en langue anglaise paraît chaque mois. Elle est en principe identique à l'édition française, et peut être obtenue aux mêmes conditions.

J. Patrognic: Derecho médico internacional - Reconocimiento de la Sociedad de la Cruz Roja de Lesotho (Circular núm. 484) - El CICR en América Latina.

Das Verbot der Folter (II) - Anerkennung des Roten Kreuzes von Lesotho (484. Rundschreiben) - Das «Manuel du Soldat» - Das «Manuel de la CroixRouge internationale » in einer neuen Auflage.

Elle est publiée chaque mois par le Comité international de la Croix-Rouge.

7, avenue de la Paix, 1211 Genève 1 (Suisse) - Compte de chèques postaux $12-1767$.

Abonnement un an: Fr. 25,-; le numéro: Fr. 2,50.

Pour les pays suivants: Algérie, Allemagne, Autriche, Belgique, Danemark, Finlande, France, Italie, Luxembourg, Maroc, Norvège, Pays-Bas, Portugal, Sarre, Suede, Tunisie, Vatican, on peut s'abonner auprès des bureaux de poste.

RÉDACTION : J.-G. LOSSIER

Seuls les textes signés par le Comité international de la Croix-Rouge engagent la responsabilité de celui-ci. 